\title{
Optical Imaging of Large Gyroid Grains in Block Copolymer Templates by Confined Crystallization
}

\author{
Raphael Dehmel, ${ }^{\dagger, \#}$ James A. Dolan, ${ }^{\dagger, \ddagger, \#}$ Yibei Gu, ${ }^{\S}$ Ulrich Wiesner, ${ }^{\S}$ Timothy D. Wilkinson,
} Jeremy J. Baumberg, ${ }^{\dagger}$ Ullrich Steiner, ${ }^{\| \odot}$ Bodo D. Wilts, ${ }^{\circledR \odot}$ and Ilja Gunkel ${ }^{*} \|_{\odot}$

${ }^{\dagger}$ Department of Physics, University of Cambridge, J.J. Thomson Avenue, Cambridge CB3 OHE, U.K.

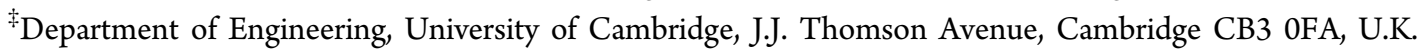

${ }^{\S}$ Department of Materials Science and Engineering, Cornell University, 214 Bard Hall, Ithaca, New York 14853-1501, United States

"Adolphe Merkle Institute, Chemin des Verdiers 4, 1700 Fribourg, Switzerland

\section{Supporting Information}

\begin{abstract}
Block copolymer (BCP) self-assembly is a promising route to manufacture functional nanomaterials for applications from nanolithography to optical metamaterials. Self-assembled cubic morphologies cannot, however, be conveniently optically characterized in the lab due to their structural isotropy. Here, the aligned crystallization behavior of a semicrystalline-amorphous polyisoprene- $b$-polystyrene- $b$ poly(ethylene oxide) (ISO) triblock terpolymer was utilized to visualize the grain structure of the cubic microphaseseparated morphology. Upon quenching from a solvent swollen state, ISO first self-assembles into an alternating

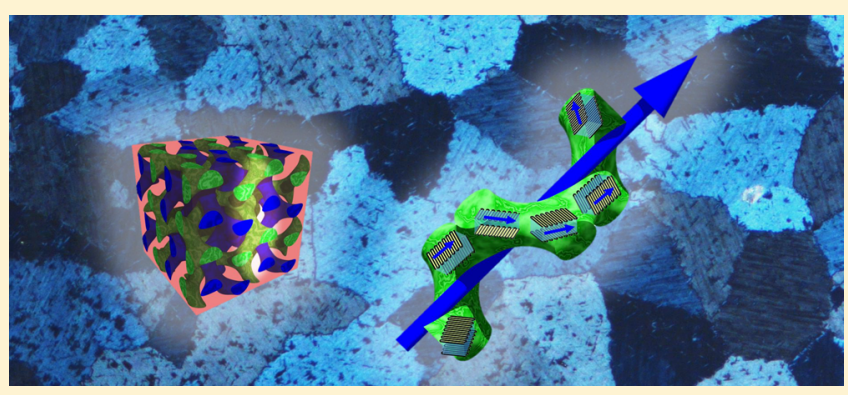
gyroid morphology, in the confinement of which the PEO crystallizes preferentially along the least tortuous pathways of the single gyroid morphology with grain sizes of hundreds of micrometers. Strikingly, the resulting anisotropic alignment of PEO crystallites gives rise to a unique optical birefringence of the alternating gyroid domains, which allows imaging of the selfassembled grain structure by optical microscopy alone. This study provides insight into polymer crystallization within a tortuous three-dimensional network and establishes a useful method for the optical visualization of cubic BCP morphologies that serve as functional nanomaterial templates.
\end{abstract}

\section{INTRODUCTION}

Block copolymer (BCP) self-assembly has recently received renewed attention because of its ability to control the structure of functional materials from the "bottom up" on the $10 \mathrm{~nm}$ length scale. ${ }^{1,2}$ Possible applications include nanolithography, ${ }^{3-5}$ antireflective coatings, ${ }^{6,7}$ optical metamaterials, ${ }^{8,9}$ photovoltaic $^{10-13}$ and battery materials. ${ }^{14-18}$ While many applications, e.g., energy materials, only require structural connectivity and high specific surface area of the nanostructured material, others, e.g., nanolithography, require detailed control of the self-assembled morphology. Although structural characterization by near-field optical and electron microscopy and small-angle scattering techniques is well established, the facile monitoring of structural evolution by optical microscopy is often impossible because of the sub-100 $\mathrm{nm}$ periodicities and structural isotropy of many self-assembled morphologies. A far simpler, optical method to characterize samples during or after processing is highly desirable as it would significantly speed up the optimization of existing fabrication procedures. This is already possible for structurally (and therefore optically) anisotropic one- and two-dimensional morphologies, e.g., aligned cylinders in solution, ${ }^{19}$ but not for structurally isotropic three-dimensional morphologies. ${ }^{20}$
One way to circumvent the lack of visibility of structurally isotropic nanostructures is to fabricate them from anisotropic materials. Aligned liquid crystals and semicrystalline polymers give rise to optical birefringence that results from subwavelength molecular arrangement. ${ }^{21}$ Semicrystalline-amorphous BCPs, i.e., those possessing both amorphous and semicrystalline blocks, provide an extension of this principle to selfassembled BCP morphologies. The present study concerns the optical imaging of grains of structurally isotropic morphologies in semicrystalline-amorphous BCPs.

Semicrystalline-amorphous BCPs also constitute a captivating class of soft condensed matter in their own right, allowing for the study of the delicate interplay between crystallization and microphase separation. ${ }^{22}$ If the crystallization temperature $T_{c}$ of the crystalline block is well below the glass transition temperature $T_{\mathrm{g}}$ of the amorphous block, the semicrystalline block undergoes confined crystallization. However, confined crystallization alone does not ensure a macroscopic alignment of crystallites within the microphase-separated morphology

\footnotetext{
Received: July 18, 2017

Revised: July 20, 2017

Published: August 7, 2017
} 
necessary for optical anisotropy. For this, the crystallite orientation with respect to the interface of the confining morphology, e.g., lamellae ${ }^{23}$ or cylinders, ${ }^{24,25}$ has to be welldefined. This orientation is a function of $T_{\mathrm{c}}$ : as $T_{\mathrm{c}}$ is increased, the long axis of the polymer chains, the crystallite $c$-axis, transitions from a random orientation to parallel and then perpendicular with respect to the interface of the polymer blocks, thereby mirroring the microphase-separated morphology. $^{26}$

Notable by their absence from studies of crystalline alignment are three-dimensional BCP network morphologies, e.g., the gyroid. The gyroid is a triply periodic isotropic structure which possesses a constant mean curvature surface. ${ }^{27}$ The convoluted gyroid channels form local subhelices of differing radii and handedness along the $\langle 111\rangle,\langle 100\rangle$, and $\langle 110\rangle$ directions which meet at triads throughout the structure. Although confined crystallization has been observed for gyroidforming semicrystalline-amorphous BCPs, ${ }^{28-30}$ no preferential orientation or alignment of the confined crystallites has been reported.

Here, we investigate the optical anisotropy of the structurally isotropic alternating gyroid morphology in thin films of a semicrystalline triblock terpolymer. We identify the physical mechanism underlying this anisotropy as a preferential alignment of confined polymer crystallites within one single gyroid network. This enables optical imaging of the gyroid BCP grain structure, thereby allowing rapid optimization of template fabrication protocols, used here to create gold gyroid optical metamaterials with exceptional long-range order.

\section{EXPERIMENTAL SECTION}

Polymer Thin Films. Polyisoprene- $b$-polysytrene- $b$-poly(ethylene oxide) (ISO) triblock terpolymers were prepared by anionic polymerization following synthesis procedures reported elsewhere. $^{31,32}$ Thin films of an $80 \mathrm{~kg} / \mathrm{mol}$ ISO with block volume fractions of $f_{\mathrm{PI}}=0.30, f_{\mathrm{PS}}=0.53$, and $f_{\mathrm{PEO}}=0.17$ were prepared atop either silicon or fluorine-doped tin oxide (FTO)-coated glass substrates. The FTO-coated glass substrates were cleaned using a piranha etch and subsequently functionalized by immersion for $15 \mathrm{~s}$ in a $0.2 \%(\mathrm{v} / \mathrm{v})$ solution of octyltrichlorosilane (Sigma-Aldrich) in anhydrous cyclohexane (Sigma-Aldrich). The silicon substrates were cleaned by exposure to an oxygen plasma (Diener MRC 100 at 100\% power) without subsequent functionalization. Thin films were spun from a $10 \%(\mathrm{w} / \mathrm{w})$ ISO solution in anhydrous anisole (Sigma-Aldrich) for $60 \mathrm{~s}$ at $1200 \mathrm{rpm}$ with an acceleration of $500 \mathrm{rpm} / \mathrm{s}$.

Solvent Vapor Annealing. ISO thin films were exposed to controlled solvent vapor atmospheres using a custom-built setup. Dry nitrogen gas was bubbled through a chloroform (Sigma-Aldrich) reservoir to take up the highest possible amount of solvent vapor. While chloroform is a good solvent for all three blocks of ISO, homopolymer swelling experiments (not shown) determine that the greatest swelling is observed for PEO, then PI, and finally PS, all with a molecular weight of $100 \mathrm{~kg} / \mathrm{mol}$. The saturated gas was then mixed with dry nitrogen to adjust the solvent concentration in the resulting gas mixture. The mixing ratio was controlled by independently varying the mass flow rate of both lines with two MKS Type 1179A mass flow controllers (100 sccm flow range; MKS PR4000B). The combined mass flow rate of nitrogen in the two lines was maintained at $20 \mathrm{sccm}$. The mass flow rate of the nitrogen bubbled through the chloroform reservoir (i.e., the proportion of saturated carrier gas) typically varied between $70 \%$ and $80 \%$ of the total mass flow rate. The temperatures of the solvent reservoir and sample were controlled using a water bath and Peltier element, respectively. The temperature of the water bath was held at $\approx 26{ }^{\circ} \mathrm{C}$ and the Peltier element at $\approx 21{ }^{\circ} \mathrm{C}$ (i.e., just below room temperature). Although possible under some annealing conditions, condensation of the solvent vapor on the sample substrate was avoided in this work by keeping the gas lines and solvent reservoir at the temperature of the bath.

Electrodeposition of Gold. The isoprene block of the microphase-separated ISO film was degraded by exposure to UV light (VWT International, $254 \mathrm{~nm}, \approx 50 \mathrm{~mW} / \mathrm{cm}^{2}$ for $2 \mathrm{~h}$ ) and subsequently removed by immersion of the film in ethanol for 10-15 min. The voided isoprene network was replicated into gold by electrodeposition using a potentiostat (Autolab PGSTAT302N) and a commercial plating solution (Metalor ECF60 with $0.5 \%$ (v/v) E3 brightener). A three-electrode cell was employed with the FTO-coated glass substrate as the working electrode, gold-coated glass as the counter electrode, and a saturated calomel reference electrode. A nucleation step (a cyclic voltammetry scan from 0 to $-1.2 \mathrm{~V}$ and back to $0 \mathrm{~V}$ at a rate of $0.05 \mathrm{~V} / \mathrm{s})$ was followed by deposition at a constant potential $(-0.8 \mathrm{~V})$ to fill the terpolymer template with gold. After deposition, the remaining PS and PEO blocks were removed by exposure of the thin film to an oxygen plasma (Diener MRC 100 at $100 \%$ power for $\approx 10$ $\min )$.

Optical Microscopy. Birefringence and linear dichroism were observed in both reflection and transmission with a standard polarizing optical microscope (Olympus BX-60). A programmable hot stage (Linkam HFS 91 with TMS 93) was used for the crystal melting experiments.

Scanning Electron Microscopy. Scanning electron micrographs were taken on a Carl Zeiss GeminiSEM operating at $5 \mathrm{kV}$.

Grazing-Incidence Wide-Angle X-ray Scattering. Grazingincidence wide-angle X-ray scattering (GIWAXS) measurements were performed at the D1 beamline of the Cornell High Energy Synchrotron Source (CHESS), Cornell University, NY, at a constant $\mathrm{X}$-ray energy of $10.67 \mathrm{keV}$. A Medoptics CCD detector was used to record the scattering images at a typical exposure time of $1-2 \mathrm{~s}$ per image. The sample-to-detector distances were calibrated with a silver behenate standard to be $102 \mathrm{~mm}$. The 2D GIWAXS data were converted into solid-angle-corrected $q_{z}$ and $q_{r}$ maps, ${ }^{33}$ where $q_{r}=\sqrt{q_{x}^{2}+q_{y}^{2}}$, and their intensity profiles were calculated using the MATLAB-based interface GIXSGUI. ${ }^{34}$

Small-Angle X-ray Scattering. Small-angle X-ray scattering (SAXS) measurements were performed at the I22 beamline of the Diamond Light Source, Didcot, UK, at a constant X-ray energy of $12.4 \mathrm{keV}$. A Pilatus $2 \mathrm{M}$ (Dectris) detector was used to record the scattering images at an exposure time of $1 \mathrm{~s}$ per image. The sample-todetector distance was calibrated with a silver behenate standard and was determined to be $5639 \mathrm{~mm}$. The 2D GISAXS scattering data were reduced using Nika, an IGOR Pro 6 (WaveMetrics Inc., Portland, OR) software package. ${ }^{35}$

Differential Scanning Calorimetry. Thermal characterization of the ISO triblock terpolymer was carried out using a Mettler Toledo DSC 2. Bulk ISO samples were loaded into aluminum DSC pans and sealed with aluminum lids. The samples were initially heated to 125 ${ }^{\circ} \mathrm{C}$, then cooled to $-80{ }^{\circ} \mathrm{C}$, and finally heated again to $125{ }^{\circ} \mathrm{C}$, all at a heating and cooling rate of $10{ }^{\circ} \mathrm{C} / \mathrm{min}$. The first heating scan was ignored when determining the glass transitions temperatures of the styrene and isoprene blocks and the melting temperature of the PEO block. The DSC measurement was repeated twice.

\section{RESULTS AND DISCUSSION}

Thin films of polyisoprene- $b$-polystyrene- $b$-poly(ethylene oxide) (ISO) were exposed to controlled amounts of nitrogen gas carrying chloroform vapor (see Experimental Section), and a solvent vapor annealing (SVA) protocol was optimized to achieve a distinct birefringent texture in the dried films. SVA is an effective means to lower the $T_{\mathrm{g}}$ of a BCP and thereby both facilitate rapid relaxation into its equilibrium morphology and improve long-range order. ${ }^{36-44}$ The textures observed in the asspun and solvent-annealed films, small spherulites and extended domains of uniform birefringence, are shown in Figure 1. Whereas the former (Figure 1a) were robust, the latter (Figure 
1b) were highly sensitive to small variations in the annealing parameters.
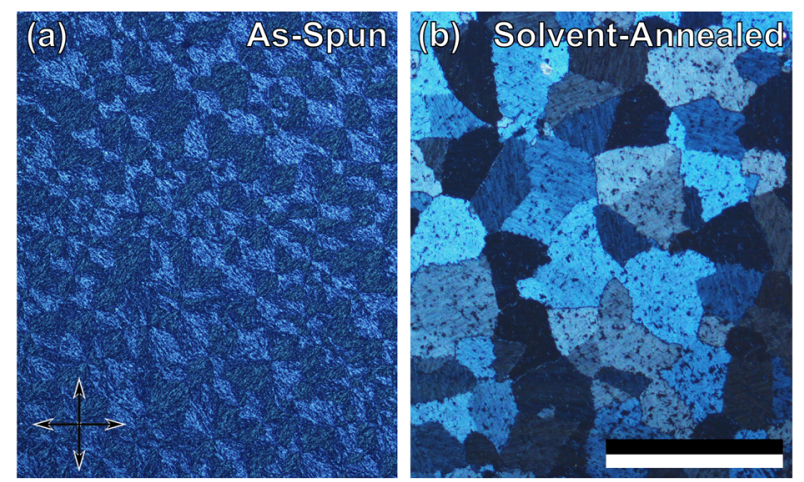

(c)

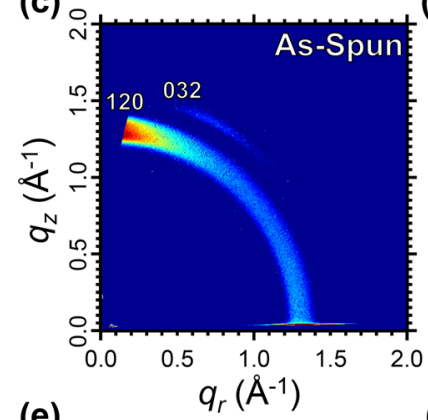

(d)

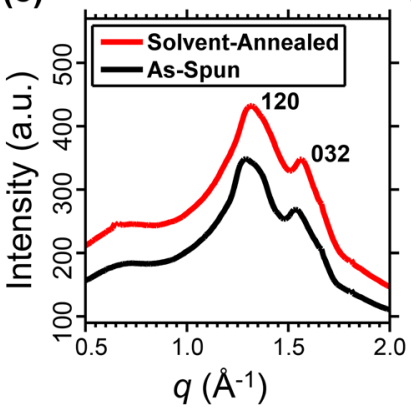

(f)
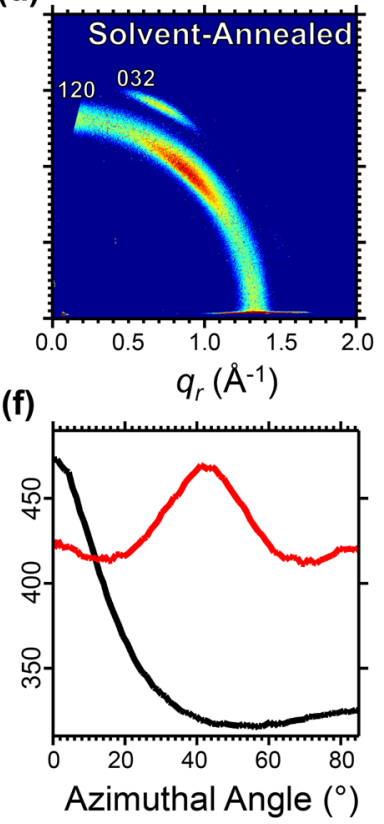

Figure 1. Optical visualization of birefringent textures of as-spun and solvent-annealed ISO thin films and their associated GIWAXS patterns. Crossed-polarization optical micrographs show (a) small spherulites in as-spun samples and (b) extended domains of uniform birefringence in solvent-annealed samples. The crossed arrows indicate the orientation of the polarizers. Scale bar: $500 \mu \mathrm{m}$ (same for a,b). (c, d) GIWAXS scattering patterns. (e) Characteristic (120) and (032) PEO reflection peaks are clearly visible in the azimuthally-averaged data for both samples. (f) The azimuthal-angle-dependent intensity distribution of the (120) signal implies an anisotropic alignment of PEO crystallites.

Melting of the birefringent textures at $T_{\mathrm{m}}^{\mathrm{PEO}} \approx 55{ }^{\circ} \mathrm{C}$ and subsequent recrystallization corroborate that the birefringence results from the semicrystalline nature of the poly(ethylene oxide) (PEO) block (Supporting Information Figure S1). Any structural anisotropy (see below) from an underlying morphology should disappear only upon heating above the $T_{\mathrm{g}}$ of polystyrene (PS), $T_{\mathrm{g}}^{\mathrm{PS}} \approx 90^{\circ} \mathrm{C} .{ }^{19}$ Both $T_{\mathrm{m}}^{\mathrm{PEO}}$ and $T_{\mathrm{g}}^{\mathrm{PS}}$ were determined by differential scanning calorimetry (DSC) (Figure S2). Semicrystalline polymers are typically positively birefringent with the extraordinary optic axis parallel to the stretched chains. They are therefore only visible under crossed polarizers when the crystallite $c$-axes have a nonzero projection onto the image plane, i.e., when the polymer chains are parallel to or tilted with respect to the substrate. The birefringent texture consisting of extended, birefringent domains implies such an alignment of the PEO crystallites over exceptionally large length scales $(\approx 100 \mu \mathrm{m})$. In an semicrystalline-amorphous $\mathrm{BCP}$, the crystalline dimensions are limited by the neighboring amorphous phase of the microphase-separated morphology $(\approx 0.1 \mu \mathrm{m})$. The birefringent domains must therefore consist of large numbers of similarly aligned PEO crystallites rather than a single macroscopic crystal. The nature of the birefringence and its impressive uniformity imply a macroscopic alignment of the constituent semicrystalline PEO.

This macroscopic alignment of PEO crystallites in the ISO thin films was further corroborated by grazing-incidence wideangle X-ray scattering (GIWAXS) measurements. Recorded scattering patterns for as-spun samples (Figure 1c) and solventannealed samples (Figure 1d) both exhibit strong peaks corresponding to the characteristic (120) and (032) reflections of PEO (Figure 1e). Note that the $q$ values of the (120) reflections of both films are 3-5\% smaller than values reported for uniaxially oriented homopolymer PEO after stretching and melt quenching. ${ }^{45}$ The slightly larger PEO unit cell sizes are probably due to complexation of PEO with residual solvent molecules, and a closer inspection of the (120) reflections reveals a bimodal crystal structure, indicating the coexistence of neat and solvent-complexed PEO crystallites (Figure S3). ${ }^{46}$ To determine the apparent crystallite size $L_{c}$ in the solventannealed films, the full width at half-maximum (fwhm) of the (120) reflection was calculated in a sector of the azimuthal angle between $40^{\circ}$ and $50^{\circ}$. Applying the Scherrer equation gives $L_{\mathrm{c}} \approx 3.4 \mathrm{~nm}$ in the $\langle 120\rangle$ direction. ${ }^{47}$ The azimuthal distributions of the (120) reflections, measured from the substrate normal, show significant anisotropy (Figure 1f). For the as-spun sample, a peak at $0^{\circ}$ is clearly visible, implying a predominant substrate-parallel orientation of the PEO crystallite $c$-axes. ${ }^{23}$ For the solvent-annealed sample, the peak at 42.3 $\pm 0.1^{\circ}\left(\right.$ fwhm $\left.=23.9^{\circ}\right)$ indicates a different preferential alignment of the semicrystalline PEO. The GIWAXS results therefore further corroborate the hypothesis that solventannealed samples consist of macroscopically aligned PEO crystallites.

As the polyisoprene (PI) phase of the microphase-separated morphology is continuous and makes contact with the conductive substrate, it may be selectively removed and replicated into metal by electrodeposition. ${ }^{8,9,48,49}$ The far greater contrast between the resulting gold network and its surrounding environment allows, after removal of the polymeric template, additional characterization of the microphaseseparated morphology by both scanning electron microscopy (SEM) and small-angle X-ray scattering (SAXS).

The morphologies of both as-spun and solvent-annealed samples on fluorine-doped tin oxide (FTO)-coated glass substrates were therefore investigated by SEM after replication of the PI phase into gold and removal of the remaining BCP (Figure 2a,b). While the as-spun sample exhibited a disordered continuous structure (Figure $2 \mathrm{a}$ ), the solvent-annealed sample showed two differently oriented continuous ordered grains (Figure 2b). Previously, it was shown that a $53 \mathrm{~kg} / \mathrm{mol}$ ISO with similar block volume fractions formed an oriented alternating gyroid phase with the $\langle 110\rangle$ direction perpendicular to the substrate. ${ }^{50}$ The $\langle 111\rangle$ and $\langle 100\rangle$ directions are then inclined by $35^{\circ}$ and $45^{\circ}$ with respect to the substrate normal, respectively. The highly uniform 6-fold (top right) and 4-fold (bottom left) pore symmetries of the two different grains in 


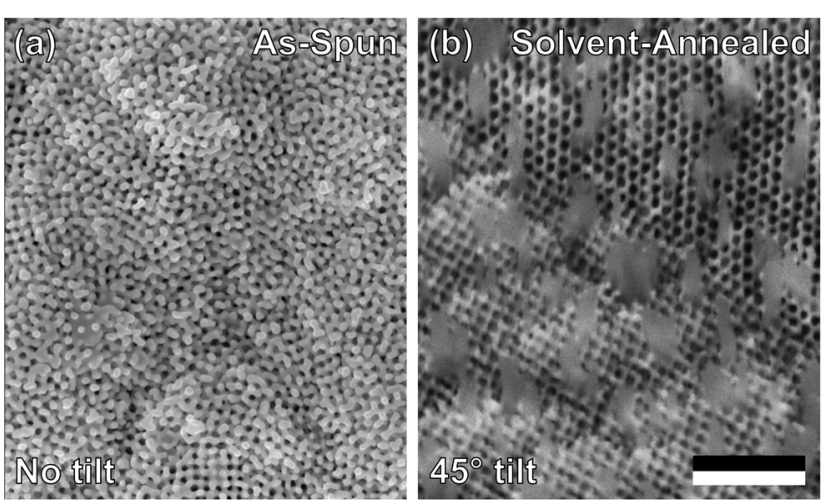

(c)

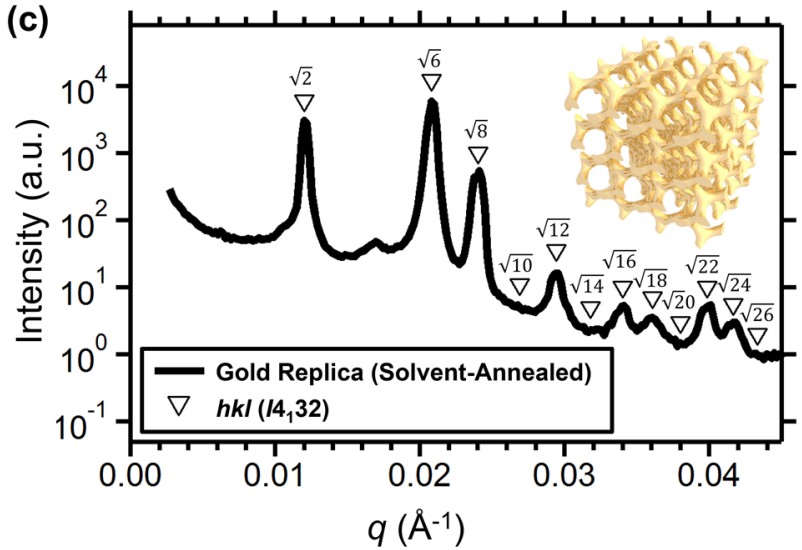

Figure 2. Scanning electron microscope (SEM) micrographs and small-angle X-ray scattering (SAXS) profile of as-spun and solventannealed ISO networks replicated into gold. (a) The gold replica of an as-spun sample exhibits a disordered continuous structure while (b) the replica of a solvent-annealed sample exhibits a regular and highly uniform array of pores, imaged under an angle of $45^{\circ}$. Two grains with different orientations are visible. The flakes on the film surface are residues from the electrodeposition procedure. Scale bar: $500 \mathrm{~nm}$. (c) Azimuthally-averaged SAXS data for a solvent-annealed film with extended birefringent domains after replication into gold indexed with the single gyroid symmetry $(I 4,32)$. Peaks are labeled with respect to the fundamental wave vector $q^{*}$.

Figure $2 \mathrm{~b}$ are both consistent with a single gyroid network, similarly oriented with the $\langle 110\rangle$ direction perpendicular to the substrate. $^{27}$

A gold replica of the solvent-annealed sample was also characterized by SAXS (Figure 2c) to further corroborate its alternating gyroid morphology. Noting that the corresponding SEM micrograph is consistent with a single gyroid morphology, the observed peaks in the line integrations of the scattering patterns may confidently be indexed and assigned to the $I 4_{1} 32$ (i.e., the single gyroid) symmetry, which corresponds to the alternating gyroid morphology of the ISO terpolymer template. Missing peaks are anticipated to result from minima in the form factor of the single gyroid (e.g., those at $\sqrt{10} q^{*}$ and $\left.\sqrt{14} q^{*}\right) .^{51}$

Gold replication of the alternating gyroid morphology creates an optical metamaterial that gives rise to linear dichroism of the sample caused by the polarization-dependent in-coupling and propagation of plasmonic modes in the subwavelength periodic metal lattice. ${ }^{8,9,49}$ Note that the polymer gyroid before metal deposition is not an optical metamaterial and its optical response is determined entirely by the nonplasmonic properties of the constituent PEO crystallites. The comparison of optical micrographs taken before and after gold replication of solvent- annealed samples and subsequent polymer removal reveals nearly identical grain patterns (Figure 3). While similar in
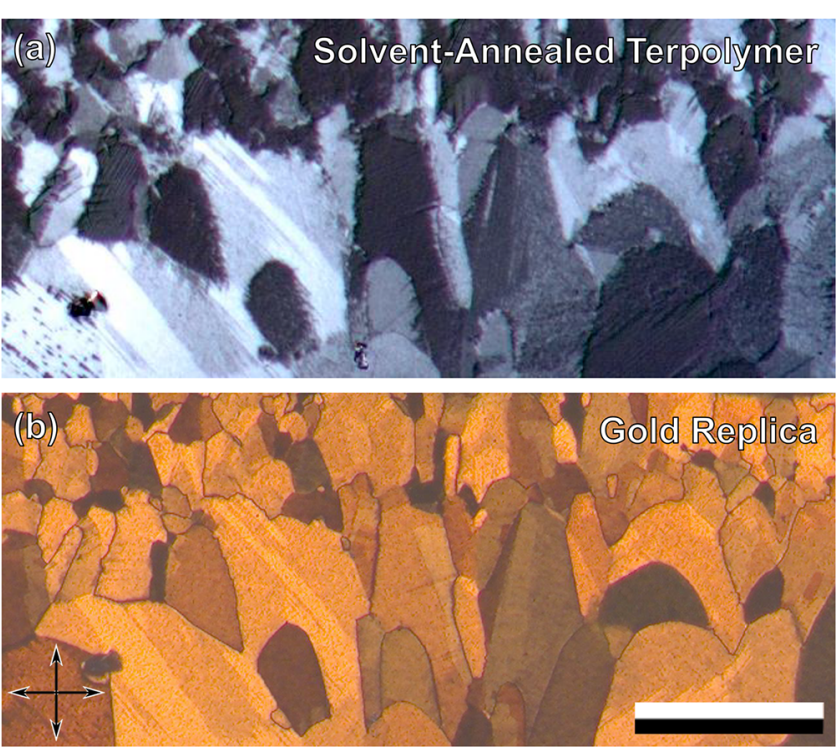

Figure 3. Birefringence and linear dichroism of a solvent-annealed ISO thin film (a) and its gold optical metamaterial replica (b). Nearly identical domain patterns are observed, indicating a correlation between the crystalline superstructure and the terpolymer morphology. Optical micrographs were taken under crossed polarizers. The crossed arrows indicate the orientation of the polarizers. Scale bar: 200 $\mu \mathrm{m}$ (same for $\mathrm{a}, \mathrm{b})$.

appearance, the birefringence (terpolymer) and linear dichroism (gold replica) of the domains have fundamentally different physical origins. In the terpolymer film (Figure 3a), birefringence arises from the preferential alignment of PEO crystallites. In contrast, the optical metamaterial (Figure $3 \mathrm{~b}$ ) shows linear dichroism due to polarization-dependent light incoupling into the gyroid morphology. ${ }^{8,27}$ To confirm that the dichroism in the replica is indeed plasmonic, the heating experiments of Figure S1 were repeated, and no change in dichroism was detected for temperatures up to $120{ }^{\circ} \mathrm{C}$. Similarly, no traces of PEO were found by GIWAXS, confirming the lack of semicrystalline PEO (not shown). The optical microscopy results before and after gold replication of solvent-annealed samples imply that the extended domains of uniform birefringence coincide precisely with individual grains of the alternating gyroid morphology. Similarly, the exceptional long-range order of the terpolymer template, used here to fabricate the gyroid optical metamaterial, bears testament to rapid and facile optimization of the template fabrication protocol based only upon optical imaging. Note that the linear dichroism of the metamaterial is indicative of the gyroid grain orientation, while the birefringence of the polymer template is determined by the local crystallite orientations.

Solvent-annealed ISO films exhibit extended birefringent domains consisting of macroscopically aligned PEO crystallites (Figure 1). The films also possess an alternating gyroid morphology with exceptional long-range order (Figure 2). There is a striking correlation between the birefringent domains formed by preferentially aligned PEO crystallites and the grains of the gyroid morphology (Figure 3). We therefore determine that PEO is both confined to and aligned by the single gyroid morphology. In this context, it is useful to distinguish between 
structural and molecular birefringence of a polymeric morphology. Structural birefringence arises from an anisotropic alignment of amorphous structural elements within a morphology, while molecular birefringence arises from the inherent molecular anisotropy of the molecules. Since cubic symmetries (such as that of the gyroid) are structurally isotropic, the optical anisotropy of the polymeric gyroid grains observed in Figure $1 \mathrm{~b}$ must arise from the molecular birefringence of PEO crystallites.

There is significant evidence to support the conclusion that the PEO undergoes confined crystallization during the SVA protocols used here. SEM (Figure $2 b$ ) and SAXS (Figure 2c) both provide strong evidence for the single gyroid morphology of the replicated PI network. In contrast, breakout crystallization would strongly favor a lamellar morphology, which is inconsistent with the observed results. ${ }^{52}$

For $T_{\mathrm{C}}^{\mathrm{PEO}}>0^{\circ}$, PEO crystallization under cylindrical confinement proceeds along the axis of the cylindrical channels, which corresponds to a perpendicular orientation of the crystallite $c$-axes with respect to the cylinder axis. ${ }^{25}$ Unlike perfectly cylindrical channels, the channel segments of a single gyroid are not straight, and their tortuosity imposes an additional constraint upon the PEO crystallization. As shown previously, the tortuosity of a pathway through the gyroid network depends on direction. ${ }^{53}$ Figure 4 illustrates the two

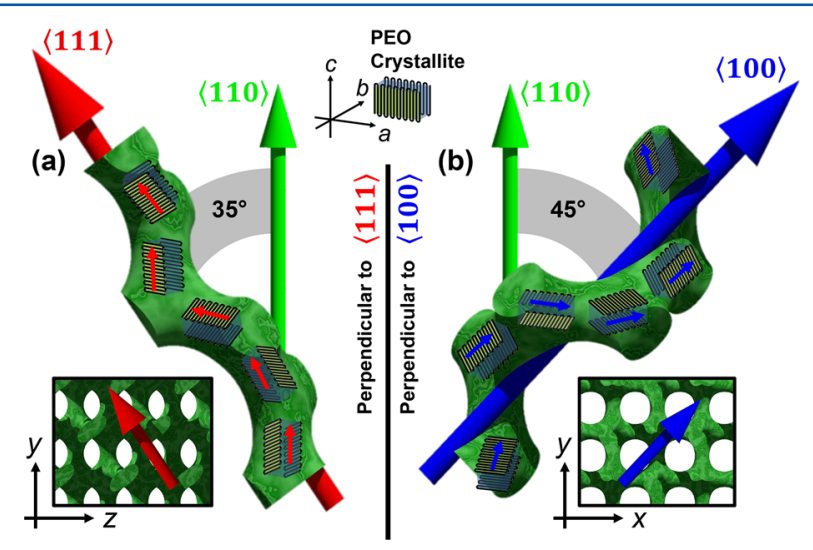

Figure 4. Proposed alignment mechanism of PEO crystallites in a single gyroid network. Details of the gyroid channels in the (a) $\langle 111\rangle$ (large red arrow) and (b) $\langle 100\rangle$ (large blue arrow) directions. The small red and blue arrows, which indicate the orientation perpendicular to the local PEO crystallite $c$-axes, follow the axis of the gyroid channels. Insets are projections of the complete gyroid network.

least tortuous pathways through the single gyroid morphology, those along the $\langle 111\rangle$ and $\langle 100\rangle$ directions, both of which form helical paths of locally cylindrical channel segments with a constant radius and which intersect with neighboring channels at $120^{\circ}$ and $90^{\circ}$, respectively. The straightness of the channels along these directions depends on the volume fill fraction of the gyroid: the larger the fill fraction, the straighter the channels. For gyroids with a fill fraction of $\approx 17 \%$, the volume fraction of the PEO block investigated here, the $\langle 111\rangle$ direction contains the straightest channels (Figure 4a); the channels along the $\langle 100\rangle$ directions are $7.5 \%$ longer and therefore slightly more tortuous (Figure $4 \mathrm{~b}$ ). ${ }^{53}$ We therefore postulate that these two directions minimize the overall free energy of the PEO crystallites or, alternatively, facilitate the kinetics of PEO crystallization. According to this hypothesis, the PEO crystallites grow preferentially along the $\langle 111\rangle$ and $\langle 100\rangle$ directions of the gyroid, with a slight preference for the shorter $\langle 111\rangle$ direction.

The macroscopic alignment of polymer crystallites within a microphase-separated morphology, observed indirectly by optical microscopy and directly by GIWAXS (Figure 1), is influenced by both the macroscopic orientation of the morphology and the microscopic orientation of the crystallites with respect to the semicrystalline-amorphous interface. Despite the remarkable long-range order of the gyroid grains presented here, they cannot be individually probed by X-ray scattering techniques, which would allow direct correlation of the alignment of the morphology with the crystallite orientation. Instead, comparisons can be made with how PEO crystallite orientations vary in other morphologies. Previous results showed that PEO in a PS- $b$-PEO BCP $\left(8.8 \mathrm{~kg} / \mathrm{mol}\right.$ PEO and $\left.24.5 \mathrm{~kg} / \mathrm{mol} \mathrm{PS} ; f_{\mathrm{PEO}}=26 \%\right)$ crystallizes with the $c$-axes aligned perpendicular to the long cylinder axes when the crystallization temperature $T_{\mathrm{C}}^{\mathrm{PEO}}$ is larger than $0{ }^{\circ} \mathrm{C} .{ }^{25}$ Given the morphological similarity of straight cylinders and gyroid channels, and the small degree of supercooling imposed here $\left(T_{\mathrm{C}}^{\mathrm{PEO}} \approx 21^{\circ} \mathrm{C}\right)$, it is likely that the gyroid channels dictate the alignment of PEO $c$-axes in a similar manner, i.e., perpendicular to the axis of the cylinder segments that make up the gyroid channels.

The ISO gyroid grains exhibit an out-of-plane $\langle 110\rangle$ orientation (Figure 4). ${ }^{8,50}$ The least tortuous $\langle 100\rangle$ and $\langle 111\rangle$ directions are correspondingly either in the plane of the substrate or angled at $45^{\circ}$ and $35^{\circ}$ with respect to the substrate normal. The helical nature of the channels in these directions will induce a significant variation of the local PEO crystallite $c$-axes perpendicular to the $\langle 111\rangle$ and $\langle 100\rangle$ directions. These variations are indicated by the small red and blue arrows in Figure 4, which follow the cylinder segments of the gyroid channels. The PEO crystallite alignment perpendicular to the channel axes with approximately equal likelihood in both the $\langle 111\rangle$ and $\langle 100\rangle$ directions should lead to broad GIWAXS peaks at azimuthal angles of $35^{\circ}$ and $45^{\circ}$. Instead, only one peak at $\approx 40^{\circ}$ is observed (Figure 1f). This single peak could arise from preferential PEO crystallization along the $\langle 111\rangle$ channels, corresponding to a $35^{\circ}$ GIWAXS peak. Alternatively, crystallization along both the $\langle 111\rangle$ and $\langle 100\rangle$ channels could lead to an overlap of two $35^{\circ}$ and $45^{\circ}$ GIWAXS peaks. The observed peak value of $42.3 \pm 0.1^{\circ}$ may indicate, in both cases, a compression of the gyroid morphology in the terpolymer film after SVA, resulting in increased out-ofplane angles. $^{12}$

The macroscopic PEO crystallite alignment is also indirectly observed by optical microscopy. Individual PEO crystallites are positively birefringent and possess an extraordinary optic axis oriented along the crystallite $c$-axes. The random orientation of these positively birefringent crystallite $c$-axes within the planes perpendicular to the local helical path of the gyroid channel is optically equivalent to negatively birefringent crystallites with their $c$-axes aligned along the same helical path. Although the orientation of these optically equivalent crystallite $c$-axes varies around the $\langle 111\rangle$ and $\langle 100\rangle$ directions, when averaged over the period of the subwavelength gyroidal spirals, any radial components of the oscillating optical axes sum to zero. The resulting effective optical axis of the crystalline superstructure is therefore aligned along the net tangential orientation of the optically equivalent crystallite $c$-axes, i.e., along the $\langle 111\rangle$ and 
$\langle 100\rangle$ directions. This results in a birefringence that varies with the in-plane orientation of the gyroid domain (Figure $1 \mathrm{~b}$ ).

The behavior of as-spun samples is entirely different than that of solvent-annealed samples. The GIWAXS pattern resulting from an as-spun sample possesses a peak at $0^{\circ}$ azimuthal angle (Figures 1a and 1f), consistent with the substrate-parallel $c$-axis orientation reported for spherulites in thin films of semicrystalline homopolymers. ${ }^{54,55}$ The disordered morphology of the as-spun samples (Figure 2a) seems to fail to impose a preferred PEO crystallization direction, resulting in small spherulites, similar to unconfined PEO films. This reinforces the crucial contribution of the gyroid morphology to the aligned crystallization observed in the solvent-annealed films.

\section{CONCLUSION}

We have studied confined PEO crystallization within a single gyroid network of a triblock terpolymer film. Our results demonstrate a preferential PEO crystallite alignment along the $\langle 111\rangle$ and $\langle 100\rangle$ directions of the single gyroid network. These directions are preferred due to the minimum tortuosity of the network in these directions, minimizing the frustration induced by the directional change of crystallization and enhancing crystallization kinetics. While observable by GIWAXS, the surprising consequence is the optical visibility of self-assembled gyroid grains which, unlike aligned cylinders or lamellae, ${ }^{19,56}$ are structurally isotropic. This visibility is unambiguously assigned to the molecular birefringence of confined and aligned PEO crystallites and is expected to be transferable to other semicrystalline copolymers that can undergo confined crystallization. ${ }^{52}$ As a result, this study not only contributes to the fundamental understanding of polymer crystallization under nanoscale confinement but also demonstrates a new tool for the study of structurally isotropic BCP morphologies that are useful templates for functional materials. Imaging of self-assembled materials typically requires either near-field optical methods or electron microscopy. Indirect methods, such as scattering techniques, may average over large sample volumes and are often cumbersome. Making use of aligned polymer crystallization therefore allows the direct, nondestructive imaging of grains in self-assembled semicrystalline-amorphous BCP films. In doing so, the fabrication of polymer templates may be rapidly optimized, as demonstrated here with the fabrication of well-ordered gold gyroid optical metamaterials.

\section{ASSOCIATED CONTENT}

\section{S Supporting Information}

The Supporting Information is available free of charge on the ACS Publications website at DOI: 10.1021/acs.macromol.7b01528. Additional data related to this publication is available at the University of Cambridge data repository (https:// doi.org/10.17863/CAM.12265).

GIWAXS profiles of ISO films, DSC of the ISO terpolymer, polarization micrographs of an ISO film during heating and cooling (PDF)

\section{AUTHOR INFORMATION}

\section{Corresponding Author}

*E-mail ilja.gunkel@unifr.ch; Ph +41 (0)26 3007398 (I.G.). ORCID

Jeremy J. Baumberg: 0000-0002-9606-9488

Ullrich Steiner: 0000-0001-5936-339X
Bodo D. Wilts: 0000-0002-2727-7128

Ilja Gunkel: 0000-0001-5738-5309

\section{Present Addresses}

R.D.: Papierfabrik Louisenthal GmbH, 83701 Gmund a.T., Germany.

Y.G.: The Dow Chemical Company, 2301 N. Brazosport Blvd, Freeport, TX 77541.

\section{Author Contributions}

${ }^{\#}$ R.D. and J.A.D. made equal contributions.

Notes

The authors declare no competing financial interest.

\section{ACKNOWLEDGMENTS}

We thank B. Heck, G. Reiter, and S. Vignolini for useful discussions. We also thank A. Sepe, D. Smilgies, and X. Sheng for their help during the experiments at CHESS. This research was supported through the Swiss National Science Foundation through grant numbers 163220 (U.S.) and 168223 (B.D.W.), the National Center of Competence in Research Bio-Inspired Materials (U.S., B.D.W, I.G.), the Adolphe Merkle Foundation (B.D.W., U.S., I.G.), the Engineering and Physical Sciences Research Council through the Cambridge NanoDTC EP/ G037221/1, EP/L027151/1, EP/N016920/1, and EP/ G060649/1 (R.D., J.A.D., J.J.B.), and ERC LINASS 320503 (J.J.B.). This project has also received funding from the European Union's Horizon 2020 research and innovation programme under the Marie Sklodowska-Curie grant agreement No 706329/cOMPoSe (I.G.). Y.G. and U.W. thank the National Science Foundation (DMR-1409105) for financial support. Part of the work was conducted at beamline D1 at the Cornell High Energy Synchrotron Source (CHESS); CHESS is supported by the NSF and NIH/NIGMS via NSF award DMR1332208. We also thank Diamond Light Source for access to beamline I22 (SM13448) that contributed to the results presented here.

\section{REFERENCES}

(1) Schacher, F. H.; Rupar, P. A.; Manners, I. Functional Block Copolymers: Nanostructured Materials with Emerging Applications. Angew. Chem., Int. Ed. 2012, 51, 7898-7921.

(2) Bates, C. M.; Bates, F. S. 50th Anniversary Perspective: Block Polymers-Pure Potential. Macromolecules 2017, 50, 3-22.

(3) Ruiz, R.; Kang, H.; Detcheverry, F. A.; Dobisz, E.; Kercher, D. S.; Albrecht, T. R.; de Pablo, J. J.; Nealey, P. F. Density Multiplication and Improved Lithography by Directed Block Copolymer Self-Assembly. Science 2008, 321, 936-939.

(4) Lin, C.-H.; Polisetty, S.; O’Brien, L.; Baruth, A.; Hillmyer, M. A.; Leighton, C.; Gladfelter, W. L. Size-Tuned ZnO Nanocrucible Arrays for Magnetic Nanodot Synthesis via Atomic Layer Deposition-Assisted Block Polymer Lithography. ACS Nano 2015, 9, 1379-1387.

(5) Bates, C. M.; Maher, M. J.; Janes, D. W.; Ellison, C. J.; Willson, C. G. Block Copolymer Lithography. Macromolecules 2014, 47, 2-12.

(6) Stefik, M.; Guldin, S.; Vignolini, S.; Wiesner, U.; Steiner, U. Block copolymer self-assembly for nanophotonics. Chem. Soc. Rev. 2015, 44, 5076-5091.

(7) Guldin, S.; Kohn, P.; Stefik, M.; Song, J.; Divitini, G.; Ecarla, F.; Ducati, C.; Wiesner, U.; Steiner, U. Self-Cleaning Antireflective Optical Coatings. Nano Lett. 2013, 13, 5329-5335.

(8) Vignolini, S.; Yufa, N. A.; Cunha, P. S.; Guldin, S.; Rushkin, I.; Stefik, M.; Hur, K.; Wiesner, U.; Baumberg, J. J.; Steiner, U. A 3D optical metamaterial made by self-assembly. Adv. Mater. 2012, 24, OP23-OP27.

(9) Salvatore, S.; Demetriadou, A.; Vignolini, S.; Oh, S. S.; Wuestner, S.; Yufa, N. A.; Stefik, M.; Wiesner, U.; Baumberg, J. J.; Hess, O.; 
Steiner, U. Tunable 3D Extended Self-Assembled Gold Metamaterials with Enhanced Light Transmission. Adv. Mater. 2013, 25, 2713-2716. (10) Dante, M.; Yang, C.; Walker, B.; Wudl, F.; Nguyen, T.-Q. SelfAssembly and Charge-Transport Properties of a PolythiopheneFullerene Triblock Copolymer. Adv. Mater. 2010, 22, 1835-1839.

(11) Mulherin, R. C.; Jung, S.; Huettner, S.; Johnson, K.; Kohn, P.; Sommer, M.; Allard, S.; Scherf, U.; Greenham, N. C. Ternary Photovoltaic Blends Incorporating an All-Conjugated Donor-Acceptor Diblock Copolymer. Nano Lett. 2011, 11, 4846-4851.

(12) Crossland, E. J. W.; Kamperman, M.; Nedelcu, M.; Ducati, C.; Wiesner, U.; Smilgies, D.-M.; Toombes, G. E. S.; Hillmyer, M. A.; Ludwigs, S.; Steiner, U.; Snaith, H. J. A Bicontinuous Double Gyroid Hybrid Solar Cell. Nano Lett. 2009, 9, 2807-2812.

(13) Segalman, R. A.; McCulloch, B.; Kirmayer, S.; Urban, J. J. Block Copolymers for Organic Optoelectronics. Macromolecules 2009, 42, 9205-9216.

(14) Javier, A. E.; Patel, S. N.; Hallinan, D. T., Jr.; Srinivasan, V.; Balsara, N. P. Simultaneous Electronic and Ionic Conduction in a Block Copolymer: Application in Lithium Battery Electrodes. Angew. Chem., Int. Ed. 2011, 50, 9848-9851.

(15) Singh, M.; Odusanya, O.; Wilmes, G. M.; Eitouni, H. B.; Gomez, E. D.; Patel, A. J.; Chen, V. L.; Park, M. J.; Fragouli, P.; Iatrou, H.; Hadjichristidis, N.; Cookson, D.; Balsara, N. P. Effect of Molecular Weight on the Mechanical and Electrical Properties of Block Copolymer Electrolytes. Macromolecules 2007, 40, 4578-4585.

(16) McIntosh, L. D.; Kubo, T.; Lodge, T. P. Morphology, Modulus, and Conductivity of a Triblock Terpolymer/Ionic Liquid Electrolyte Membrane. Macromolecules 2014, 47, 1090-1098.

(17) Inceoglu, S.; Rojas, A. A.; Devaux, D.; Chen, X. C.; Stone, G. M.; Balsara, N. P. Morphology-Conductivity Relationship of SingleIon-Conducting Block Copolymer Electrolytes for Lithium Batteries. ACS Macro Lett. 2014, 3, 510-514.

(18) Young, W.-S.; Kuan, W.-F.; Epps, T. H. Block Copolymer Electrolytes for Rechargeable Lithium Batteries. J. Polym. Sci., Part B: Polym. Phys. 2014, 52, 1-16.

(19) Chastek, T. Q.; Lodge, T. P. Measurement of Gyroid Single Grain Growth Rates in Block Copolymer Solutions. Macromolecules 2003, 36, 7672-7680.

(20) Meuler, A. J.; Hillmyer, M. A.; Bates, F. S. Ordered Network Mesostructures in Block Polymer Materials. Macromolecules 2009, 42, $7221-7250$.

(21) Collings, P.; Hird, M. Introduction to Liquid Crystals: Chemistry and Physics; Liquid Crystals Book Series; CRC Press: 1997.

(22) He, W.-N.; Xu, J.-T. Crystallization assisted self-assembly of semicrystalline block copolymers. Prog. Polym. Sci. 2012, 37, 13501400.

(23) Zhu, L.; Cheng, S. Z. D.; Calhoun, B. H.; Ge, Q.; Quirk, R. P.; Thomas, E. L.; Hsiao, B. S.; Yeh, F.; Lotz, B. Crystallization temperature-dependent crystal orientations within nanoscale confined lamellae of a self-assembled crystalline-amorphous diblock copolymer. J. Am. Chem. Soc. 2000, 122, 5957-5967.

(24) Huang, P.; Zhu, L.; Cheng, S. Z. D.; Ge, Q.; Quirk, R. P.; Thomas, E. L.; Lotz, B.; Hsiao, B. S.; Liu, L.; Yeh, F. Crystal Orientation Changes in Two-Dimensionally Confined Nanocylinders in a Poly(ethylene oxide)-b-polystyrene/Polystyrene Blend. Macromolecules 2001, 34, 6649-6657.

(25) Huang, P.; Guo, Y.; Quirk, R. P.; Ruan, J.; Lotz, B.; Thomas, E. L.; Hsiao, B. S.; Avila-Orta, C. A.; Sics, I.; Cheng, S. Z. D. Comparison of poly(ethylene oxide) crystal orientations and crystallization behaviors in nano-confined cylinders constructed by a poly(ethylene oxide)-b-polystyrene diblock copolymer and a blend of poly(ethylene oxide)-b-polystyrene and polystyrene. Polymer 2006, 47, 5457-5466.

(26) Strobl, G. The Physics of Polymers: Concepts for Understanding Their Structures and Behavior; Springer: 2007.

(27) Dolan, J. A.; Wilts, B. D.; Vignolini, S.; Baumberg, J.; Steiner, U.; Wilkinson, T. D. Optical properties of gyroid structured materials: from photonic crystals to metamaterials. Adv. Opt. Mater. 2015, 3, 1232 .
(28) Sun, L.; Zhu, L.; Ge, Q.; Quirk, R. P.; Xue, C.; Cheng, S. Z. D.; Hsiao, B. S.; Avila-Orta, C. A.; Sics, I.; Cantino, M. E. Comparison of crystallization kinetics in various nanoconfined geometries. Polymer 2004, 45, 2931-2939.

(29) Loo, Y.-L.; Register, R. A.; Ryan, A. J.; Dee, G. T. Polymer Crystallization Confined in One, Two, or Three Dimensions. Macromolecules 2001, 34, 8968-8977.

(30) Weimann, P. A.; Hajduk, D. A.; Chu, C.; Chaffin, K. A.; Brodil, J. C.; Bates, F. S. Crystallization of tethered polyethylene in confined geometries. J. Polym. Sci., Part B: Polym. Phys. 1999, 37, 2053-2068.

(31) Bailey, T. S.; Pham, H. D.; Bates, F. S. Morphological Behavior Bridging the Symmetric $\mathrm{AB}$ and $\mathrm{ABC}$ States in the Poly(styrene-bisoprene-b-ethylene oxide) Triblock Copolymer System. Macromolecules 2001, 34, 6994-7008.

(32) Bailey, T. S.; Hardy, C. M.; Epps, T. H.; Bates, F. S. A noncubic triply periodic network morphology in poly (isoprene-b-styrene-bethylene oxide) triblock copolymers. Macromolecules 2002, 35, 70077017.

(33) Müller-Buschbaum, P. The Active Layer Morphology of Organic Solar Cells Probed with Grazing Incidence Scattering Techniques. Adv. Mater. 2014, 26, 7692-7709.

(34) Jiang, Z. GIXSGUI: a MATLAB toolbox for grazing-incidence $\mathrm{X}$-ray scattering data visualization and reduction, and indexing of buried three-dimensional periodic nanostructured films. J. Appl. Crystallogr. 2015, 48, 917-926.

(35) Ilavsky, J. Nika: software for two-dimensional data reduction. J. Appl. Crystallogr. 2012, 45, 324-328.

(36) Müller-Buschbaum, P. GISAXS and GISANS as metrology technique for understanding the 3D morphology of block copolymer thin films. Eur. Polym. J. 2016, 81, 470-493.

(37) Shelton, C. K.; Epps, T. H. Block copolymer thin films: Characterizing nanostructure evolution with in situ X-ray and neutron scattering. Polymer 2016, 105, 545-561.

(38) Gu, X.; Gunkel, I.; Hexemer, A.; Gu, W.; Russell, T. P. An in situ grazing incidence $\mathrm{X}$-ray scattering study of block copolymer thin films during solvent vapor annealing. Adv. Mater. 2014, 26, 273-281.

(39) Baruth, A.; Seo, M.; Lin, C. H.; Walster, K.; Shankar, A.; Hillmyer, M. A.; Leighton, C. Optimization of long-range order in solvent vapor annealed poly(styrene)-block-poly(lactide) thin films for nanolithography. ACS Appl. Mater. Interfaces 2014, 6, 13770-13781.

(40) Gu, X.; Gunkel, I.; Hexemer, A.; Russell, T. P. Controlling Domain Spacing and Grain Size in Cylindrical Block Copolymer Thin Films by Means of Thermal and Solvent Vapor Annealing. Macromolecules 2016, 49, 3373-3381.

(41) Sinturel, C.; Vayer, M.; Morris, M.; Hillmyer, M. A. Solvent Vapor Annealing of Block Polymer Thin Films. Macromolecules 2013, 46, 5399-5415.

(42) Posselt, D.; Zhang, J.; Smilgies, D.-M.; Berezkin, A.; Potemkin, I. I.; Papadakis, C. M. Restructuring in Block Copolymer Thin Films: InSitu GISAXS Investigations During Solvent Vapor Annealing. Prog. Polym. Sci. 2017, 66, 80-115.

(43) Paik, M. Y.; Bosworth, J. K.; Smilges, D.-M.; Schwartz, E. L.; Andre, X.; Ober, C. K. Reversible morphology control in block copolymer films via solvent vapor processing: An in situ GISAXS study. Macromolecules 2010, 43, 4253-4260.

(44) Chavis, M. A.; Smilgies, D. M.; Wiesner, U. B.; Ober, C. K. Widely tunable morphologies in block copolymer thin films through solvent vapor annealing using mixtures of selective solvents. Adv. Funct. Mater. 2015, 25, 3057-3065.

(45) Takahashi, Y.; Tadokoro, H. Structural Studies of Polyethers, $(-(\mathrm{CH} 2) \mathrm{m}-\mathrm{O}-)$ n. X. Crystal Structure of Poly(ethylene oxide). Macromolecules 1973, 6, 672-675.

(46) Paternostre, L.; Damman, P.; Dosiere, M. Morphology and crystal structure of the poly(ethylene oxide)-hydroquinone molecular complex. J. Polym. Sci., Part B: Polym. Phys. 1999, 37, 1197-1208.

(47) Smilgies, D.-M. Scherrer grain-size analysis adapted to grazingincidence scattering with area detectors. J. Appl. Crystallogr. 2009, 42, $1030-1034$. 
(48) Salvatore, S. Optical Metamaterials by Block Copolymer SelfAssembly (Springer Theses); Springer: Berlin, 2014.

(49) Dolan, J. A.; Saba, M.; Dehmel, R.; Gunkel, I.; Gu, Y.; Wiesner, U.; Hess, O.; Wilkinson, T. D.; Baumberg, J. J.; Steiner, U.; Wilts, B. D. Gyroid Optical Metamaterials: Calculating the Effective Permittivity of Multidomain Samples. ACS Photonics 2016, 3, 1888-1896.

(50) Stefik, M.; Wang, S.; Hovden, R.; Sai, H.; Tate, M. W.; Muller, D. A.; Steiner, U.; Gruner, S. M.; Wiesner, U. Networked and chiral nanocomposites from $\mathrm{ABC}$ triblock terpolymer coassembly with transition metal oxide nanoparticles. J. Mater. Chem. 2012, 22, 10781087.

(51) Saranathan, V.; Osuji, C. O.; Mochrie, S. G. J.; Noh, H.; Narayanan, S.; et al. Structure, function, and self-assembly of single network gyroid (I4132) photonic crystals in butterfly wing scales. Proc. Natl. Acad. Sci. U. S. A. 2010, 107, 11676-11681.

(52) Loo, Y.-L.; Register, R. A.; Ryan, A. J. Modes of Crystallization in Block Copolymer Microdomains: Breakout, Templated, and Confined. Macromolecules 2002, 35, 2365-2374.

(53) Scherer, M. R. J.; Cunha, P. M. S.; Steiner, U. Labyrinth-Induced Faceted Electrochemical Growth. Adv. Mater. 2014, 26, 2403-2407.

(54) Michell, R. M.; Müller, A. J. Confined crystallization of polymeric materials. Prog. Polym. Sci. 2016, 54-55, 183-213.

(55) Liu, Y.-X.; Chen, E.-Q. Polymer crystallization of ultrathin films on solid substrates. Coord. Chem. Rev. 2010, 254, 1011-1037.

(56) Chastek, T. Q.; Lodge, T. P. Twinning and Growth Kinetics of Lamellar Grains in a Diblock Copolymer Solution. J. Polym. Sci, Part B: Polym. Phys. 2005, 43, 405-412. 